\title{
Data collection and processing with a direct electron detector
}

Johan Hattne ${ }^{a, *}$, Michael W. Martynowycz ${ }^{a}$ \& Tamir Gonen ${ }^{a}$

${ }^{a}$ Howard Hughes Medical Institute, Departments of Biological Chemistry and Physiology, David Geffen School of Medicine, University of California, Los Angeles, Los Angeles, CA 90095, USA

*hattne@ucla.edu

Microcrystal electron diffraction (MicroED) permits us eful diffraction data collection from nanosized crystals. MicroED has been successful where other techniques are not applicable, in some cases yielding structures to sub-atomic resolution by $a b$ initio methods. Usually, MicroED involves a continuously rotating sample probed by high-energy electrons while the diffraction pattern is read out on a fast, shutterless camera. Even though electrons interact favorably with matter, small crystals of large proteins require an efficient electron detector to be accurately recorded. This is particularly true for radiation-sensitive specimen, for which signal strength cannot be enhanced by increasing the exposure without damaging the sample. This presentation explores the link between crystal size, radiation damage, and camera performance. Using the Falcon III direct electron detector, which was designed for imaging in electron cryo-microscopy (cryo-EM), it is shown that data can be collected faster and with significantly lower exposure than previously reported. 